\title{
Role of Plasmodium berghei ookinete surface and oocyst capsule protein, a novel oocyst capsule-associated protein, in ookinete motility
}

\author{
Kazuhiko Nakayama' , Yuta Kimura', Yu Kitahara' ${ }^{1}$ Akira Soga ${ }^{2}$, Asako Haraguchi' , Jun Hakozaki ${ }^{1}$, \\ Makoto Sugiyama ${ }^{3}$, Kodai Kusakisako', Shinya Fukumoto ${ }^{2}$ and Hiromi Ikadai ${ }^{*}$
}

\begin{abstract}
Background: Plasmodium sp., which causes malaria, must first develop in mosquitoes before being transmitted. Upon ingesting infected blood, gametes form in the mosquito lumen, followed by fertilization and differentiation of the resulting zygotes into motile ookinetes. Within $24 \mathrm{~h}$ of blood ingestion, these ookinetes traverse mosquito epithelial cells and lodge below the midgut basal lamina, where they differentiate into sessile oocysts that are protected by a capsule.
\end{abstract}

Methods: We identified an ookinete surface and oocyst capsule protein (OSCP) that is involved in ookinete motility as well as oocyst capsule formation.

Results: We found that knockout of OSCP in parasite decreases ookinete gliding motility and gradually reduces the number of oocysts. On day 15 after blood ingestion, the oocyst wall was significantly thinner. Moreover, adding anti-OSCP antibodies decreased the gliding speed of wild-type ookinetes in vitro. Adding anti-OSCP antibodies to an infected blood meal also resulted in decreased oocyst formation.

Conclusion: These findings may be useful for the development of a transmission-blocking tool for malaria.

Keywords: Plasmodium berghei, Ookinete, Oocyst, Knock out parasite, Transmission block

\section{Background}

Malaria is among the world's deadliest infectious diseases. In 2018, over 200 million people were infected and over 400,000 people died [1]. While significant advances have been made in the fight against malaria, progress has slowed in recent years mainly because of the prevalence of drug-resistant parasites and insecticide-resistant mosquitoes. To conquer malaria, it is vital to understand the biology of parasite transmission better and develop new tools for fighting the disease.

*Correspondence: ikadai@vmas.kitasato-u.ac.jp

${ }^{1}$ Laboratory of Veterinary Parasitology, School of Veterinary Medicine, Kitasato University, Towada, Aomori 034-8628, Japan

Full list of author information is available at the end of the article
After the mosquito ingests an infected blood meal, parasites form gametes, followed by fertilization and differentiation of the resulting zygotes into motile ookinetes that traverse the midgut epithelium and lodge below the midgut basal lamina. It takes $>20 \mathrm{~h}$ from blood ingestion until the ookinete arrives at the midgut basal lamina. During this time, the parasites are attacked by both the mammalian and mosquito immune systems [2]. As a result, parasite numbers decrease by over a thousand fold, creating a potent bottleneck $[3,4]$. The ookinete then differentiates into an oocyst and produces a capsule that helps it avoid the mosquito's immune response. Within the capsule, many sporozoites are formed and released into the hemolymph, thus increasing parasite numbers by several thousand fold [5].

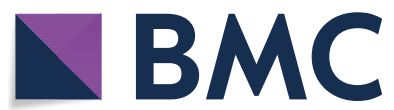

(c) The Author(s) 2021. This article is licensed under a Creative Commons Attribution 4.0 International License, which permits use, sharing, adaptation, distribution and reproduction in any medium or format, as long as you give appropriate credit to the original author(s) and the source, provide a link to the Creative Commons licence, and indicate if changes were made. The images or other third party material in this article are included in the article's Creative Commons licence, unless indicated otherwise in a credit line to the material. If material is not included in the article's Creative Commons licence and your intended use is not permitted by statutory regulation or exceeds the permitted use, you will need to obtain permission directly from the copyright holder. To view a copy of this licence, visit http://creativecommons.org/licenses/by/4.0/. The Creative Commons Public Domain Dedication waiver (http://creativecommons.org/publicdomain/zero/1.0/) applies to the data made available in this article, unless otherwise stated in a credit line to the data. 
The mosquito mounts a strong immune response against the invading parasite [6, 7], and Plasmodium sp. produces a number of proteins to protect itself, some of which are expressed on the ookinete's surface. The functions of P25/P28, P47, guanylate cyclase $\beta$ (GC $\beta$ ), putative secreted ookinete surface protein (PSOP25), Plasmodium invasion of mosquito midgut screen candidate 2 (PIMMS2), and PIMMS43 surface proteins have been discussed in existing literature [2, 8-10]. P25/P28, P47, and PIMMS43 are mainly expressed to protect the ookinete from the mosquito's immune defense, while PIMMS2 is involved in protecting the ookinete while traversing the midgut cells. On the other hand, GC $\beta$ is mainly involved in the ookinete's movement ability, and PSOP25 functions in ookinete maturation. Among the proteins that accumulate in the microneme of the ookinete, the functions of Plasmodium perforin-like protein 3-5 (PPLP3-5), secreted ookinete adhesive protein (SOAP), circumsporozoite- and TRAP-related protein (CTRP), cell-traversal protein for ookinetes and sporozoites (CelTOS), and chitinase have also been described [2, 13-22]. PPLP3-5, SOAP, and CelTOS aid in traversal of the midgut. CTRP is involved in the ookinete's movement ability, while chitinase assists in traversal of the peritrophic matrix (PM), a chitin-containing layer surrounding the blood bolus.

The oocyst capsule is composed of mosquito-derived proteins, including laminin, matrix metalloprotease 1 (MMP1), and lysozyme c-1 (LYSC1) [23], and parasitederived proteins such as Plasmodium berghei oocyst capsule protein 380 (PbCap380) and oocyst capsule-associated protein 93 of Plasmodium berghei (PbCap93) [2325]. Knockout of the PbCap380 or PbCap93 genes results in decreased oocyst and sporozoite numbers [24, 25].

We focused on the transformation in the early steps of capsule formation as the ookinete differentiates into the oocyst. In this study, we investigated the functions of the high molecular weight $(494 \mathrm{kDa})$ ookinete surface and oocyst capsule protein (OSCP: PBANKA_1025100) from PlasmoDB (https://plasmodb.org/plasmo/app/). The OSCP gene was selected based on its expression on ookinete surface and oocyst capsule. Therefore, OSCP was expected to play a critical role in ookinete and oocyst stages. In this study, we characterized the role of OSCP in the ookinete stage and oocyst stage. Finally, we present a new candidate vaccine antigen with transmission-blocking properties.

\section{Methods}

\section{Parasites, mice, and mosquitoes}

For $P$. berghei infections, 6- to 8-week-old male BALB/c mice (SLC, Japan) were infected with either wild-type
(WT) P. berghei (ANKA strain) or P. berghei (ANKA strain), which constitutively expresses GFP [26].

Anopheles stephensi (STE2 strain) mosquitoes were maintained at $27{ }^{\circ} \mathrm{C}$ and $80 \%$ relative humidity with a $14 / 10 \mathrm{~h}$ light/dark cycle in an insectary and fed $10 \%(\mathrm{w} / \mathrm{v})$ sucrose solution.

For the mosquito infection experiments, P. bergheiinfected mice having ten exflagellations per $10^{4} \mathrm{RBCs}$ or more were used. In the mosquito-to-mouse infection experiment, we used mosquitoes infected with WT and knockout (KO) parasites (25 days after the mosquitoes had ingested the infected blood meal).

\section{Construction of OSCP-KO parasites}

Procedures were as previously reported [24, 27], using PCR amplification with primers OSCP-F1 (5'-CAA AAC AAT GTG GAT TTG TG-3) and OSCP-R1 (5'-GGT TTT CAT TTT CCC TAA AAT C-3) and P. berghei genomic DNA as a template. The gene was cloned into the pCR-BluntII-TOPO vector (Thermo Fisher Scientific), resulting in the plasmid pOSCP. Subsequently, pOSCP was digested using Acc I. The digested pOSCP was inserted into the hdhfr expression cassette [28]. pOSCP $(10 \mu \mathrm{g})$ was linearized with Xho I and electroporated into cultured $P$. berghei schizonts using Nucleofector II (Lonza, Basel, Switzerland). Transfected parasites were intravenously injected into male $\mathrm{BALB} / \mathrm{c}$ mice that were then treated with pyrimethamine $(70 \mu \mathrm{g} / \mathrm{ml}) 24 \mathrm{~h}$ later via drinking water.

PCRs with the following primer combinations were performed to detect the presence of recombinant parasites. T1: OSCP-F2 (5'-CCA TAC CTT CAA GAT TAG ATG AC-3) with hDHFR-shDR (5'-CTG TTA TAA TTA TGT TGT CTC TTC-3), T2: hDHFR-shDF (5'-CGA AAA GAA TTA AGC TTA ACT C-3') with OSCPR3 (5'-GCA GAT CCG TCC GTT TAA C-3'), and T3: OSCP-F2 with OSCP-R2.

To analyze OSCP expression during oocyst stage, using the Trizol reagent (Thermo Fisher Scientific, Waltham, MA, USA), total RNA was isolated from the mosquito at 10 days after infection. cDNA was synthesized using the ReverTra Ace kit (Toyobo, Osaka, Japan). PCR was performed using following primers OSCP-F3 (5'-TCG AGA TGG ATG CAA AGA CTA GCA G-3) and OSCP-R3 (5'GAT TCA CTG AAG GCT CAG GTT TAC C-3').

\section{Anti-OSCP antibody preparation and purification}

Rabbit polyclonal antibodies were raised against the following OSCP protein domains: $\alpha 1$ (amino acids 926-939) and $\alpha 2$ (mixed amino acids 1789-1802 and 3103-3116) (Eurofins Genomics Inc., Tokyo, Japan) (Fig. 1a). We utilized the epitope selection service offered by Eurofins Genomics Inc. (Tokyo, Japan) for epitope selection. The 
a

\begin{tabular}{lllll} 
Hydrophobic & \multicolumn{4}{c}{ Hydropilic } \\
\cline { 2 - 5 } & & alfa-1 Ab & alfa-2 $\mathrm{Ab}$ & alfa-2 Ab \\
\hline
\end{tabular}

b

\section{Illustration of Ookinete}

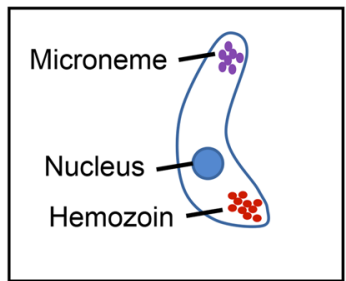

PFA fixation TritonX-100 treatment

DIC

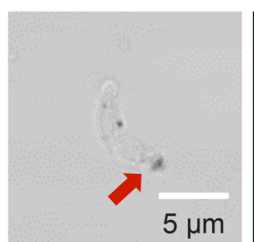

$5 \mu \mathrm{m}$

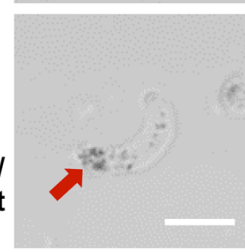

no TritonX-100 treatment

PFA fixation

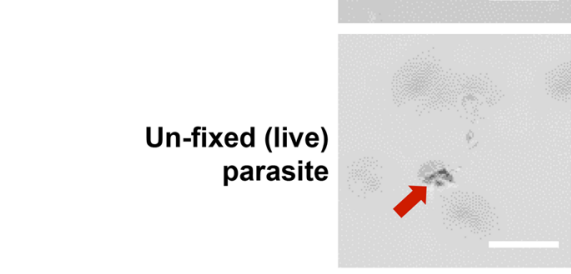

Un-fixed (live) parasite w/o Anti-OSCP

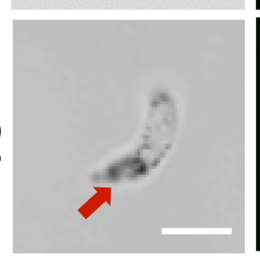

Hemozoin

\section{Anti-GFPI DAPI}
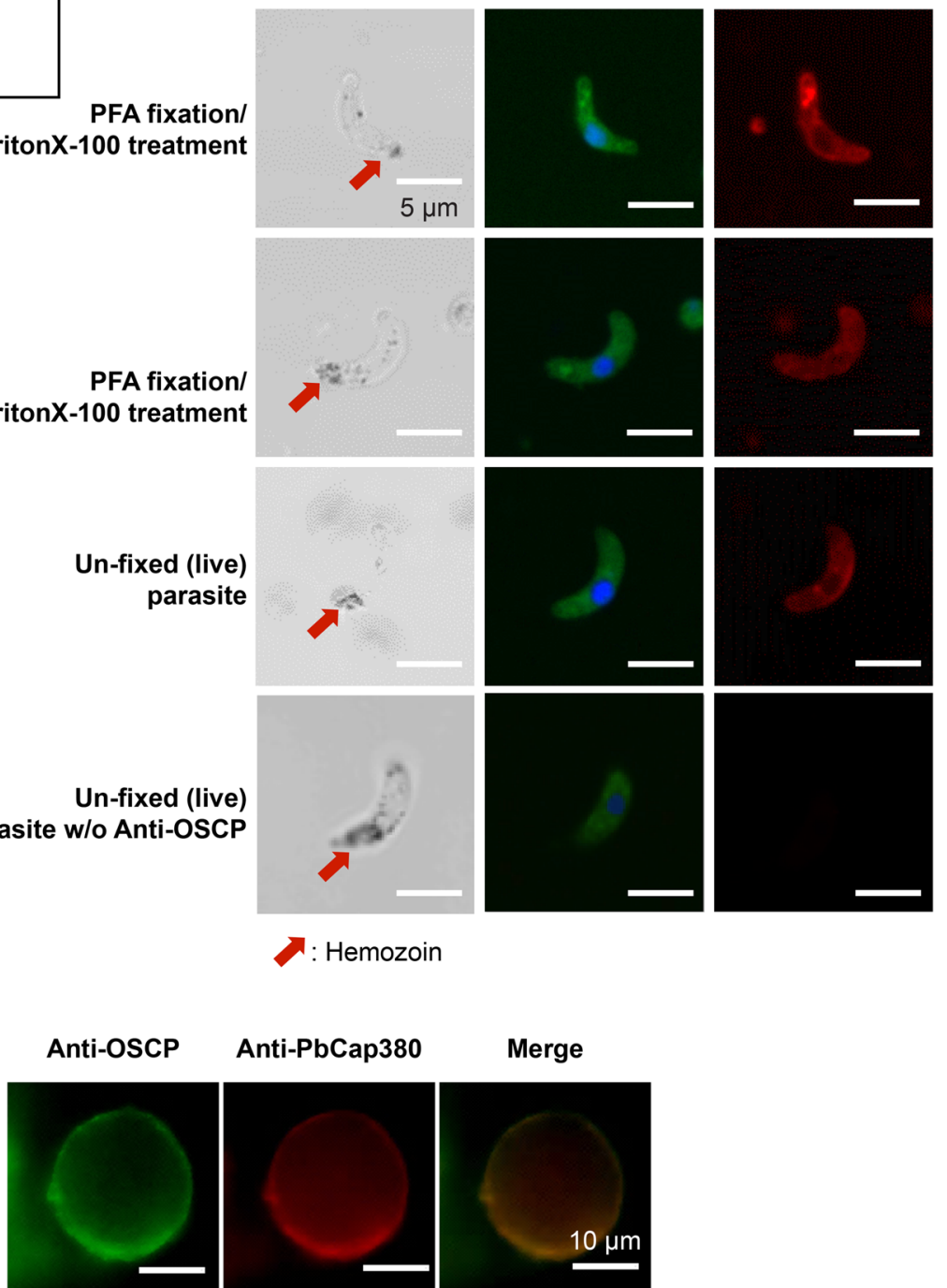

Merge

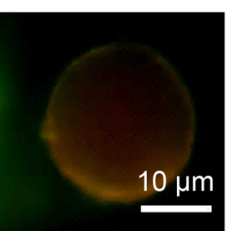

Fig. 1 OSCP is expressed in the ookinete and oocyst stages. a Schematic representation of the OSCP amino acid sequence. Polyclonal antibodies were prepared against peptide-comprising amino acids 926 to 939 (anti-OSCP a-1); a mixture of peptides and amino acids 1,789-1,802 plus 3,103-3,116 (anti-OSCP a-2) antibodies is shown. $\mathbf{b}$ Immunofluorescence analysis of OSCP expression by ookinetes. OSCP localized to the ookinete surface. The ookinete parasite was labeled twice with antibodies against GFP (green: center panel) and OSCP (red: right panel), stained with the DAPI nuclear stain (blue), and showing differential interference contrast (DIC: left panel), as indicated. Negative control was performed as only anti-GFP antibody incubation on unfixed (live) parasites (w/o Anti-OSCP). Scale-bars: $5 \mu \mathrm{m}$. c OSCP is localized to the oocyst capsule. Images of an oocyst 15 days after the blood meal 
OSCP amino acid sequence was divided into three segments on the basis of length. Among these, $\alpha 1$ domain in the first segment had the highest antigenicity. For the other two segments, antibodies against the highly antigenic regions of each were mixed to form $\alpha 2$. Antibodies were purified by saturated ammonium sulfate solution and Ab-Rapid SPiN EX (ProteNova, Japan). Ab-Rapid SPiN EX was performed following the manual until equilibration occurred. The purified antibodies were designated as anti-OSCP $\alpha 1$ and $\alpha 2$ antibodies. As a control, we also prepared non-immune rabbit antibodies by processing non-immune rabbit blood serum in the same manner.

\section{Blood-stage parasite and exflagellation analysis}

Parasitemia and gametocyte sex ratio were determined by preparing blood smear specimens from infected mice, fixing these with $100 \%$ methanol and then performing Giemsa staining. Exflagellation of male gametocytes was quantified as previously described [23, 29]. Briefly, $2 \mu \mathrm{l}$ of gametocyte-infected blood was obtained from the tail vein and mixed immediately with $38 \mu \mathrm{l}$ of the complete ookinete culture medium (OKM: RPMI 1640 medium (Gibco, USA) with 20\% heat-inactivated fetal bovine serum (Sigma-Aldrich, USA). The mixture was placed under a coverslip at room temperature (RT), and $5 \mathrm{~min}$ later, exflagellation centers were counted over the next 10 min using a phase contrast microscope. The number of exflagellation centers $/ 10^{4} \mathrm{RBCs}$ was measured per $8 \mu \mathrm{l}$ using a Thomas hemocytometer (Nippon Rinsho Kikai Kogyo Co., Ltd., Japan).

\section{Ookinete culture and purification}

Blood from mice with exflagellation of $10 / 10^{4}$ RBCs was obtained by heart puncture. Ten volumes of OKM was added to the blood and cultured for $24 \mathrm{~h}$ at $19{ }^{\circ} \mathrm{C}$. The size and number of ookinetes was measured by taking a smear from the culture solution. Ookinetes were purified using a MidiMACS separator system (Miltenyi Biotec, Germany) as previously described [30]. A total of $3 \mathrm{ml}$ culture was passed through three times before removing the column from the magnet. The ookinetes were recovered by passing $5 \mathrm{ml}$ of OKM through the column. The purified ookinetes were centrifuged at $1,000 \times g 10 \mathrm{~min}$ at $4{ }^{\circ} \mathrm{C}$ and then washed three times with PBS.

\section{Immunofluorescent assays (IFAs)}

Cultured ookinetes were placed for $30 \mathrm{~min}$ at $4{ }^{\circ} \mathrm{C}$ on a MAS-coated adhesive slide glass (Matsunami, Japan), fixed with $4 \%$ paraformaldehyde (PFA)/PBS at RT for $10 \mathrm{~min}$, and then washed three times with PBS for $5 \mathrm{~min}$ each time. After incubating for $10 \mathrm{~min}$ with $0.1 \mathrm{M}$ glycine (Wako, Japan), they were washed three times with
PBS for 5 min each time. Ookinetes were permeabilized for $10 \mathrm{~min}$ with $0.2 \%$ TritonX-100/PBS and washed with PBS for $10 \mathrm{~min}$. No TritonX-100 was added when observing OSCP surface localization. Blocking was performed for $1 \mathrm{~h}$ at RT using 1\% BSA/PBS. The primary antibody, anti-OSCP serum ( $\alpha 1$ to $\alpha 2$ ratio $1: 1)$ diluted 800 -fold with PBS, was incubated for $1 \mathrm{~h}$ at RT and then washed three times with PBS for 5 min each. Non-immunized serum was used as negative control. Incubation with the secondary antibody, Alexa Fluor ${ }^{\circledR} 488$ goat anti-rabbit IgG $(\mathrm{H}+\mathrm{L})$ (Thermo Fisher) diluted 1000-fold with PBS, was for $1 \mathrm{~h}$ at RT followed by three washes with PBS for 5 min each time. The parasites were mounted in SlowFade ${ }^{\circledR}$ Diamond Antifade Mountant with DAPI (Molecular probes, USA) and observed using an Eclipse E600 (Nikon, Japan) fluorescence microscope. The oocysts were observed by dissecting mosquitoes on day 15 after ingesting an infected blood meal, and staining was performed. Methanol fixation was performed, and after air-drying, the oocysts were treated with Image-iT FX Signal Enhancer solution (Thermo Fisher) for $30 \mathrm{~min}$ at $37^{\circ} \mathrm{C}$. After PBS washing, 400-fold-diluted anti-OSCP serum was added as the primary antibody and allowed to react for $2 \mathrm{~h}$ at $4{ }^{\circ} \mathrm{C}$. After three PBS washes, Alexa Fluor ${ }^{\circledR} 488$ goat anti-rabbit IgG $(\mathrm{H}+\mathrm{L})$ secondary antibody (Thermo Fisher) was added and allowed to react for $1 \mathrm{~h}$ at $4{ }^{\circ} \mathrm{C}$. After three PBS washes, 1000-fold-diluted anti-PbCap380 rabbit serum was added as the primary antibody and allowed to react for $2 \mathrm{~h}$ at $4{ }^{\circ} \mathrm{C}$. As the secondary antibody, Alexa Fluor ${ }^{\circledR} 568$ goat anti-rabbit IgG $(\mathrm{H}+\mathrm{L})$ (Thermo Fisher) was added and allowed to react for $1 \mathrm{~h}$ at $4{ }^{\circ} \mathrm{C}$. After three PBS washes and mounting, observation was performed with an Eclipse E600 (Nikon) fluorescence microscope.

\section{Ookinete locomotion}

Ookinete movement was investigated by mixing ookinetes with Matrigel (Corning, USA) and then measuring displacement speed [10, 31]. Purified ookinete suspension and Matrigel were mixed in equal amounts, and then $15 \mu \mathrm{l}$ drops were applied to glass slides. Glass covers $(18 \times 24 \mathrm{~mm})$ were placed on top, and the specimens were left to stand for $20 \mathrm{~min}$ on a ThermoPlate (NHP$45 \mathrm{~N}$; Nissin, Japan) at $19^{\circ} \mathrm{C}$. Next, a digital microscope (KH-8700; Hirox, Japan) was used to observe ookinete movement for $2 \mathrm{~min}$. When imaging 10-min movement trajectories, WT and $\mathrm{KO}$ parasites were incubated for 30 min at $4{ }^{\circ} \mathrm{C}$ with MitoBright LT Red (Dojindo, Japan) diluted 1000-fold with PBS. Centrifugation was performed at $1000 \times g$ for $10 \mathrm{~min}$ at $4{ }^{\circ} \mathrm{C}$ and then washed with PBS. This process was performed twice. Then, as stated above, the specimens were mixed with Matrigel and left to stand. They were then observed and imaged 
with IX83 CellSens (Olympus, Japan). In the antibody reaction experiment, antibodies diluted with PBS were added to the purified ookinete suspension and mixed with an identical amount of Matrigel before undergoing the same process. Non-immune rabbit antibodies were used as the negative control.

\section{Oocyst number and size}

Midgut oocyst numbers were counted using a Leica M205 FA (Leica, Germany) after staining the infected midguts with $0.5 \%$ mercurochrome/distilled water for $5 \mathrm{~min}$ and then washing with PBS for $5 \mathrm{~min}$ [32]. The midguts were also imaged with Leica Application Suite X (Leica, Germany). We randomly selected 60 oocysts from the imaged midguts and used Image J to measure oocyst size.

The ratio of normal to deformed oocysts was observed in mosquitoes on days 6 and 7 after the blood meal. Oocysts with an internal vacuole were considered deformed.

\section{Transmission-blocking (TB) experiment using antibodies}

$\mathrm{BALB} / \mathrm{c}$ mice were infected with GFP parasites by intraperitoneal injection of $10^{6}$ parasites. Mice that exhibited exflagellation of $10 / 10^{4}$ RBCs or more 5 days after infection were used for the experiments. In total, 50 female mosquitoes (Pre-group) were allowed to feed on the infected mouse for $15 \mathrm{~min}$. This was followed by intravenous injection of $200 \mu \mathrm{g}$ of anti-OSCP antibodies $(\alpha 1: \alpha 2=1: 1)$. After $5 \mathrm{~min}$, another 50 mosquitoes (Post group) were allowed to feed for $15 \mathrm{~min}$ on the same mouse. Oocysts were counted 2 or 10 days later. For the TB experiment assayed 2 days after the blood meal, oocyst numbers were counted again after washing the guts with PBS for 1 min to remove any attached ookinetes.

\section{Transmission electron microscopy}

On days 17 after the infected blood meal, mosquito midguts were fixed in $2.5 \%$ glutaraldehyde and $2 \%$ paraformaldehyde in $0.05 \mathrm{M}$ sodium cacodylate buffer (pH 7.4) at $4{ }^{\circ} \mathrm{C}$ for $2 \mathrm{~h}$. After rinsing, samples were post-fixed at RT in buffered $1 \%$ osmium tetroxide for $2 \mathrm{~h}$ and then dehydrated in ethanol and propylene oxide series and embedded in epoxy resin. Ultrathin sections were cut using an Ultracut N (Reichert-Nissei, Tokyo, Japan) and stained using uranyl acetate followed by lead citrate. Sections were examined using an H-7650 transmission electron microscope (Hitachi Ltd., Tokyo, Japan).

\section{Statistical analysis}

Welch's t-test was used to perform comparisons between groups (parasitemia, gametocytemia, number of ookinete, ookinete speed, oocyst size). The Mann-Whitney $U$ test was used to compare the number of oocysts/ midgut. All analyses were performed with GraphPad Prism software (GraphPad, San Diego, CA, USA).

\section{Results \\ OSCP is expressed in ookinetes and is a component of the oocyst capsule}

A PlasmoDB search performed for proteins expressed on the $P$. berghei ookinete surface and that are also part of the oocyst capsule led to the identification of OSCP (PBANKA_1025100). OSCP is predicted to have 4236 amino acids and has a molecular weight of $494 \mathrm{kDa}$. It has a predicted transmembrane domain in amino acids 97-119 (Fig. 1a). OSCP has orthologs with PF3D7_1417600 (amino acid homology: 60.8\%) of P. falciparum, PKNH_1340400 (amino acid homology: 30.8\%) of P. knowlesi, and PVP01_1331300 (amino acid homology: 33.1\%) of $P$. vivax.

To investigate the localization of OSCP expression, we labeled ookinetes and oocysts with anti-OSCP antibodies using immunofluorescence assays (IFAs). These IFAs indicate whether OSCP is present in ookinetes.

The IFA images comparing ookinetes fixed with PFA and TritonX-100 treatment, fixed with PFA and no TritonX-100 treatment, and unfixed (live) parasites showed that OSCP was expressed on the ookinete surface (Fig. 1b). PbCap380 is a surface protein of the oocyst capsule [24], and the IFAs of day 15 oocysts using anti-OSCP and anti-PbCap380 antibodies indicated that OSCP colocalizes with PbCap380 (Fig. 1c).

\section{OSCP is not required for asexual growth}

As illustrated in Fig. 2a, we obtained $\mathrm{KO}$ parasites that were confirmed by PCR (Fig. 2b) and Southern blotting (Fig. 2c). Semi- quantitative RT-PCR verified that mRNA was not transcribed (Fig. 2d). The growth of WT and KO parasites in mice was indistinguishable (Fig. 2e), as was the gametocyte sex ratio (Fig. 2f). These results suggested that OSCP does not play an important role in the bloodstage development of the parasite.

\section{OSCP is involved in ookinete locomotion}

We then investigated OSCP function in mosquito stages. There was no difference between the number of WT and $\mathrm{KO}$ ookinetes formed (Fig. 3a), and no differences were noted in ookinete size (Fig. 3b), indicating that OSCP is not involved in ookinete development or differentiation. Comparison of ookinete gliding on glass slides revealed that $\mathrm{KO}$ ookinete motility is significantly lower than WT ookinetes (Fig. 3c). Observations of ookinete trajectories over $10 \mathrm{~min}$ revealed that the number of turns performed by the $\mathrm{KO}$ ookinetes is significantly lower than that of 


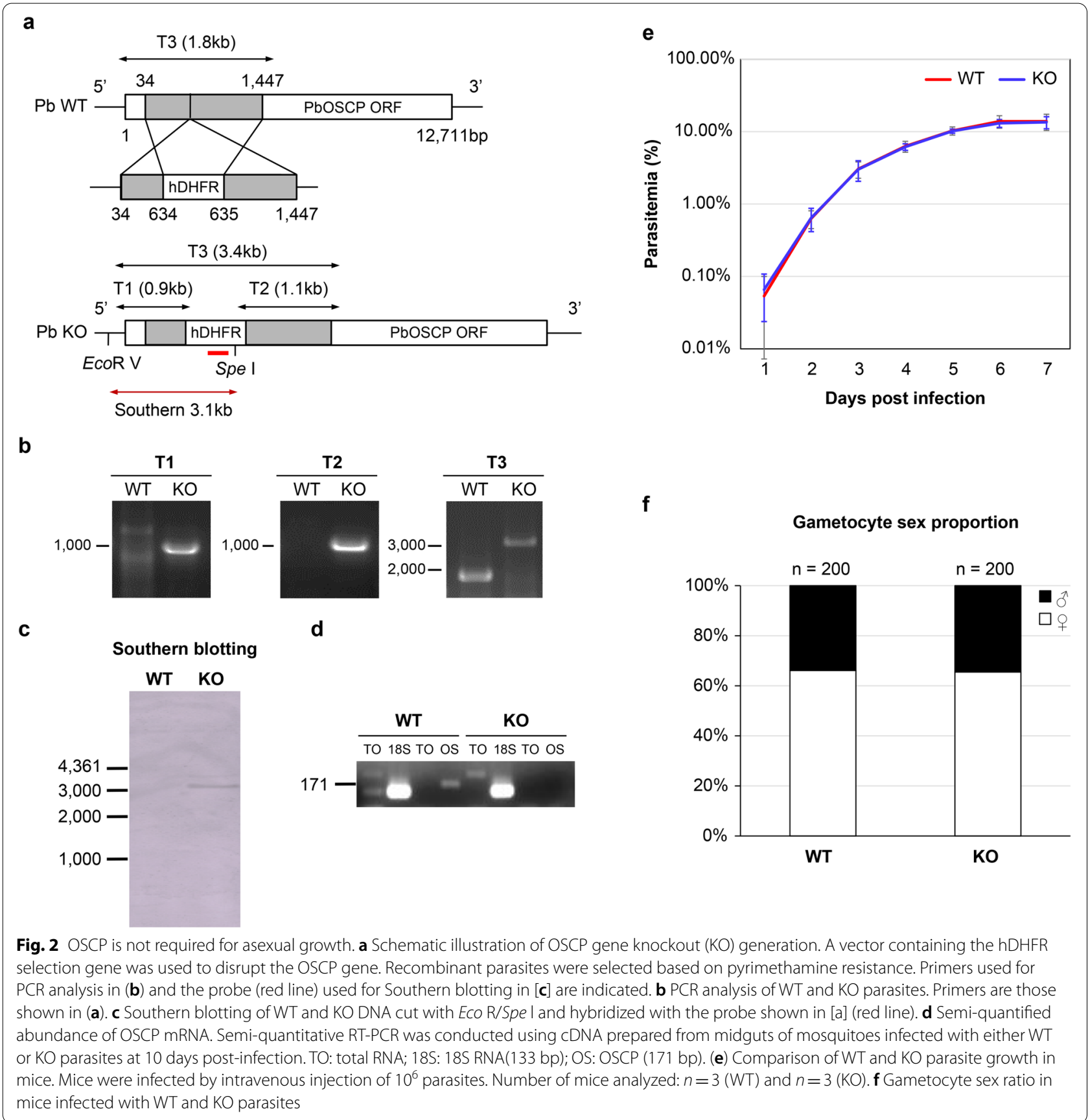

WT ookinetes (Fig. 3d, e). This holds true even after adjusting for the lower $\mathrm{KO}$ ookinete locomotion speed (Fig. 3e).

\section{OSCP is involved in oocyst capsule formation and maintenance}

We compared WT and $\mathrm{KO}$ parasite infection rates and the number of oocysts formed at 5,9 , and 14 days after ingestion of an infectious blood meal. The number of oocysts formed was significantly reduced in $\mathrm{KO}$ infections compared to WT infections at the three time points measured (Fig. 4a). Moreover, on days 9 and 14, $\mathrm{KO}$-oocyst diameter was significantly smaller than of WT oocysts (Fig. 4b). Transmission electron microscopy revealed that on day $17, \mathrm{KO}$ oocysts exhibited wall thinning and indistinguishable internal structures. Sporozoites in $\mathrm{KO}$ oocysts differed in size and cell construction compared to sporozoites in WT oocysts. While some 
a

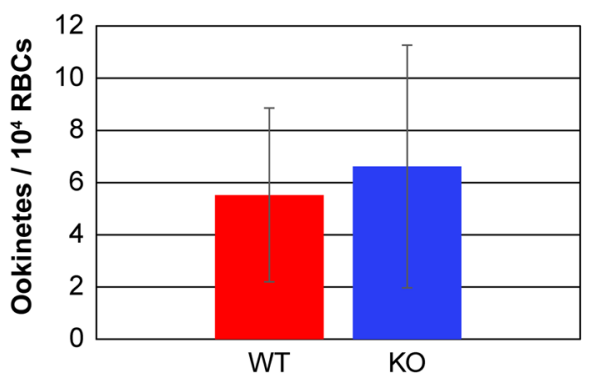

b
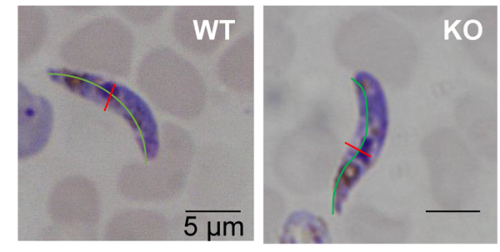

\begin{tabular}{ccc}
\hline Size $(\boldsymbol{\mu m})$ & WT & KO \\
\hline Length & $25.5 \pm 2.4$ & $25.3 \pm 4.0$ \\
Width & $4.3 \pm 0.7$ & $4.1 \pm 0.8$ \\
\hline
\end{tabular}

C

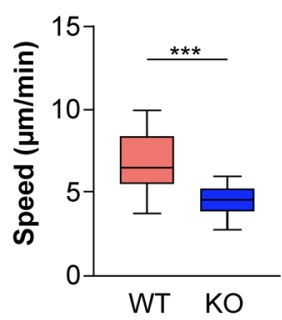

d
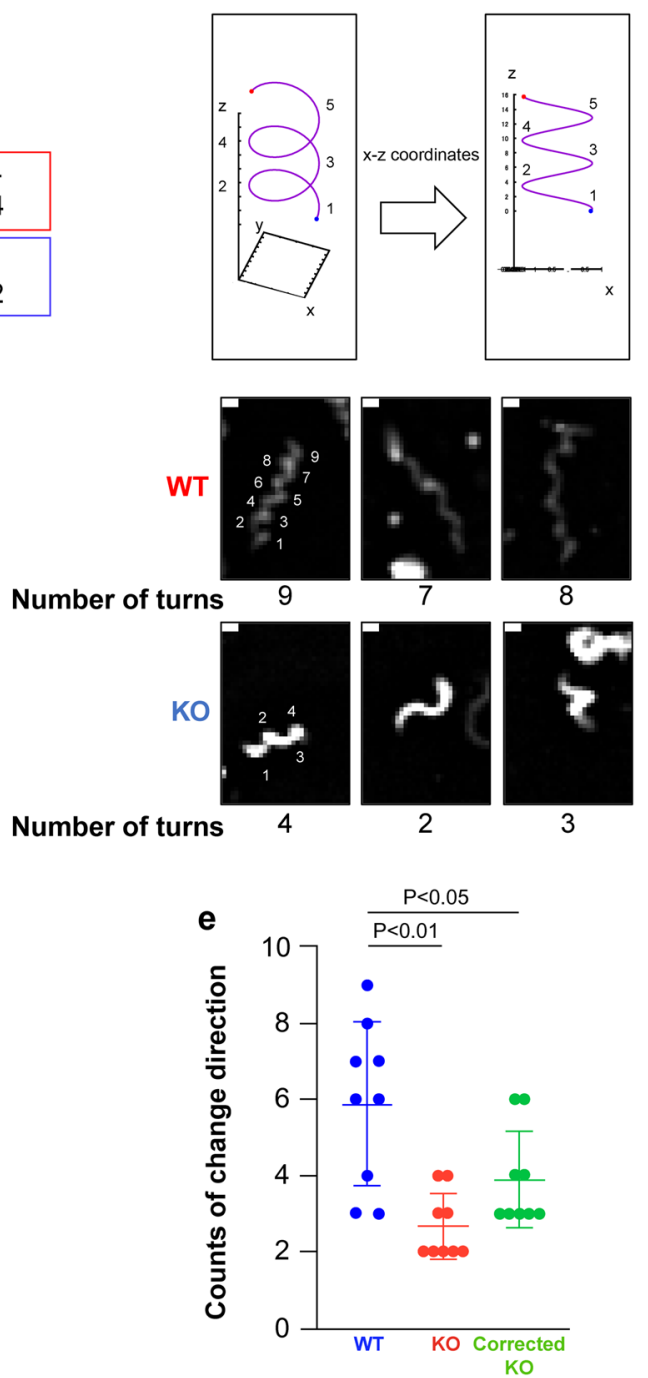

Fig. 3 OSCP is involved in ookinete locomotion. a WT and KO ookinete formation in vitro. b Comparison of ookinete dimensions. Green line: length, red line: width. Number of ookinete analyzed: $n=20$ (WT) and $n=20$ (KO). c Comparison of ookinete gliding speed. Data pooled from 20 measurements for each WT and KO parasites. Welch's t-test: ${ }^{* *} P<0.001 \mathbf{d}$ Upper panels: Schematic diagram of spiral movement. Each turn around a spiral axis is comprised of two directional changes. Lower panels: examples of trajectories over $10 \mathrm{~min}$. e Comparison of WT and KO ookinete number of turns. Corrected KO is WT ookinete speed/KO ookinete speed $(6.74 / 4.53=1.49$; panel c) multiplied by number of KO ookinete turns. The number of $\mathrm{KO}$ ookinete turns is significantly lower regardless of correction. Welch's t-test: ${ }^{*} P<0.05{ }^{* *} P<0.01$.

sporozoites of WT were observed outside of oocyst, sporozoites of $\mathrm{KO}$ were not fully matured in oocysts on day 17 (Fig. 4c). Furthermore, mercurochrome staining also showed a smaller proportion of normal oocysts (Fig. 4d). These results suggest that OSCP is involved in oocyst wall formation and maintenance. When mosquitoes were allowed to feed on naive mice 25 days after an infectious blood meal, they transmitted both WT and $\mathrm{KO}$ parasites, suggesting that OSCP is not essential for sporozoite function (data not shown).

\section{Anti-OSCP antibodies inhibit transmission to mosquitoes}

We investigated whether anti-OSCP- $\alpha 1$ and $-\alpha 2$ against epitopes presumed to be exposed to the parasite surface (Fig. 1a) can inhibit parasite function and transmission. Addition of the $\alpha 1$ antibody significantly decreased ookinete speed (Fig. 5a), while the addition of the $\alpha 2$ antibody did not affect speed (Fig. 5b).

To investigate whether a decrease in the speed of ookinete affects oocyst formation in mosquitoes, we passively immunized mice by intravenously injecting them with an 


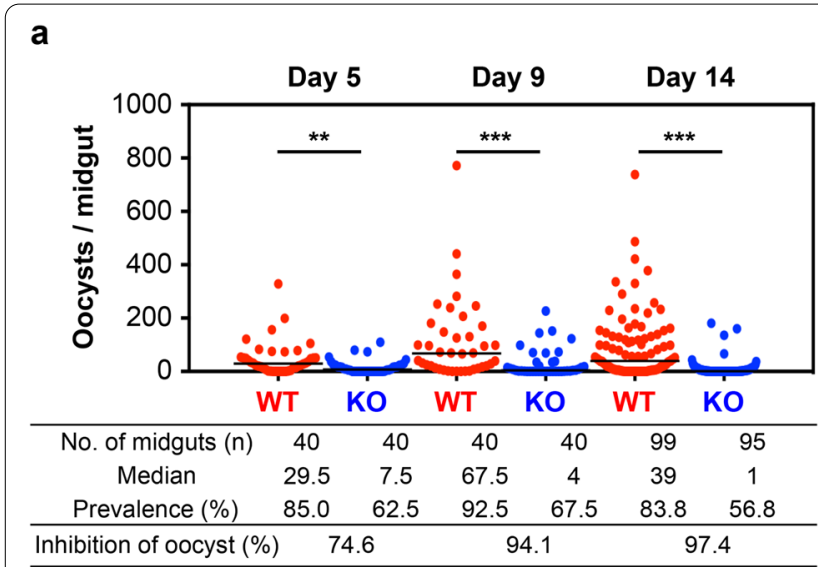

b

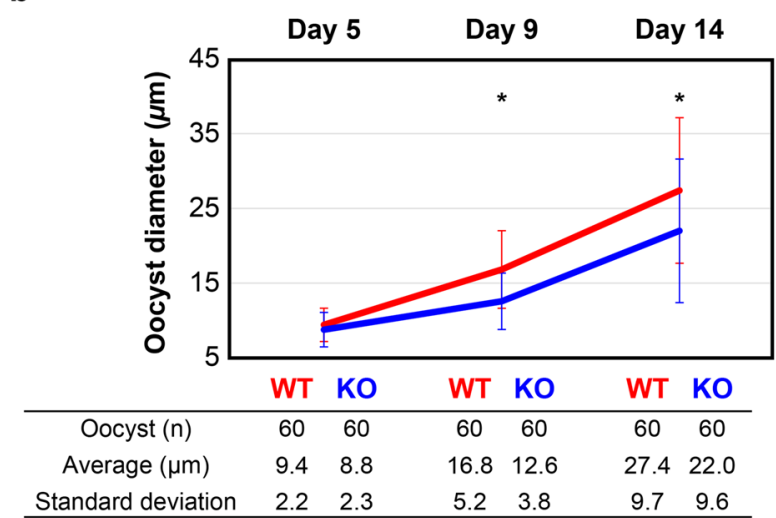

C
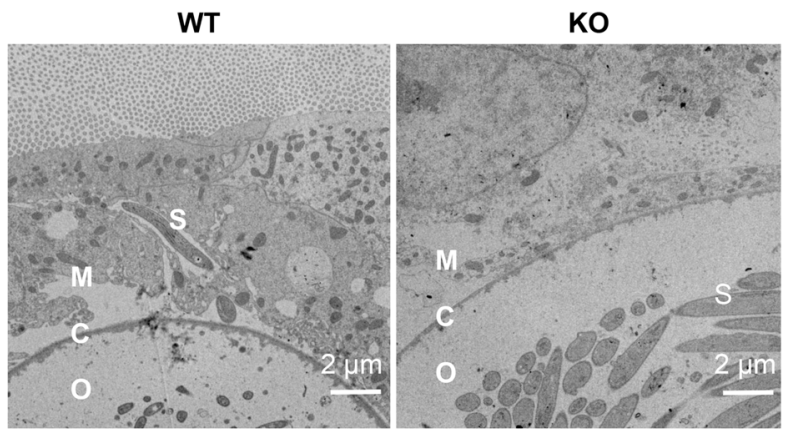

d
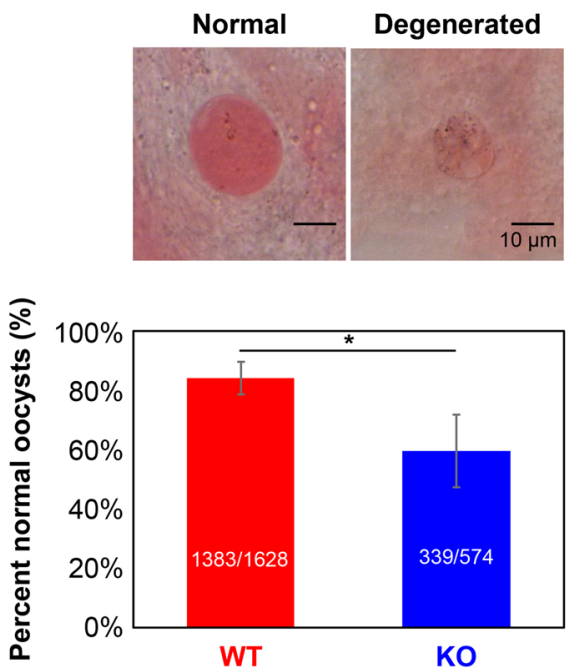

Fig. 4 OSCP is involved in oocyst wall formation and maintenance. a Number of oocysts per midgut at 5, 9, and 14 days after an infected blood meal. The horizontal bars show the median value. The inhibition of oocyst number was calculated using (1-KO-oocyst median number/WT-oocyst median number) $\times 100$. Number of independent experiments: day 5 and day 9 in duplicate and day 14 in triplicate. Mann-Whitney $U$ test:

${ }^{*} P<0.01,{ }^{* * *} P<0.001$. b Oocyst diameter per midgut at days 5, 9, and 14. Welch's t-test: ${ }^{*} P<0.01$. c Transmission electron microscopic images of oocysts at day 17. O: oocyst; C: oocyst capsule; M: mosquito tissue; S: sporozoite. d Upper panels (mercurochrome staining): examples of normal and degenerated oocysts. Lower panel: Proportion of WT and KO normal oocysts at day 6-7. Mann-Whitney $U$ test: ${ }^{*} P<0.05$.

equal mixture of $\alpha 1$ and $\alpha 2$ anti-OSCP antibodies. Antibody injection into mice imitates the immune response against OSCP antigens. Mosquitoes that fed on the blood of the same mouse prior to antibody injection served as controls. The antibodies showed an inhibitory effect on oocyst formation on day 2 after blood feeding (Fig. 5c), indicating that the antibodies act at the stage of ookinete entry into midgut cells. The experiments in Fig. $5 \mathrm{~d}$ were performed to support the data (Fig. 5c) associated with the decrease in the number of oocysts due to the decrease in the speed of ookinete movement.

\section{Discussion}

In this study, we investigated OSCP, a high molecular weight protein expressed in both the ookinete membrane and oocyst capsule. IFAs demonstrated that OSCP is expressed on the ookinete surface and also inside ookinetes after permeabilization. In OSCP-KO parasites, although no major differences were observed in ookinete growth and formation, movement speed decreased and the number of oocysts that formed was markedly lower. Thus, knocking out OSCP affected ookinete gliding. The PM represents a physical barrier between the fed blood and the midgut epithelia found in mosquito. The PM is an extracellular mesh composed of chitin, sugars, and protein [33]. When ookinetes pass through the blood as it is being ingested, because of the PM in the midgut space and midgut cells, they move forward more efficiently in a spiral trajectory instead of a straight-line trajectory, as in the case of an arrow [33]. Therefore, ookinetes move in a spiral manner from the midgut space to below the basal lamina of midgut cell. A previous in vivo experiment of mosquito midgut tissue and a previous in vitro experiment using Matrigel confirm these findings [3335]. Ookinetes that move in a linear fashion are likely to lose significant amounts of energy and become trapped 


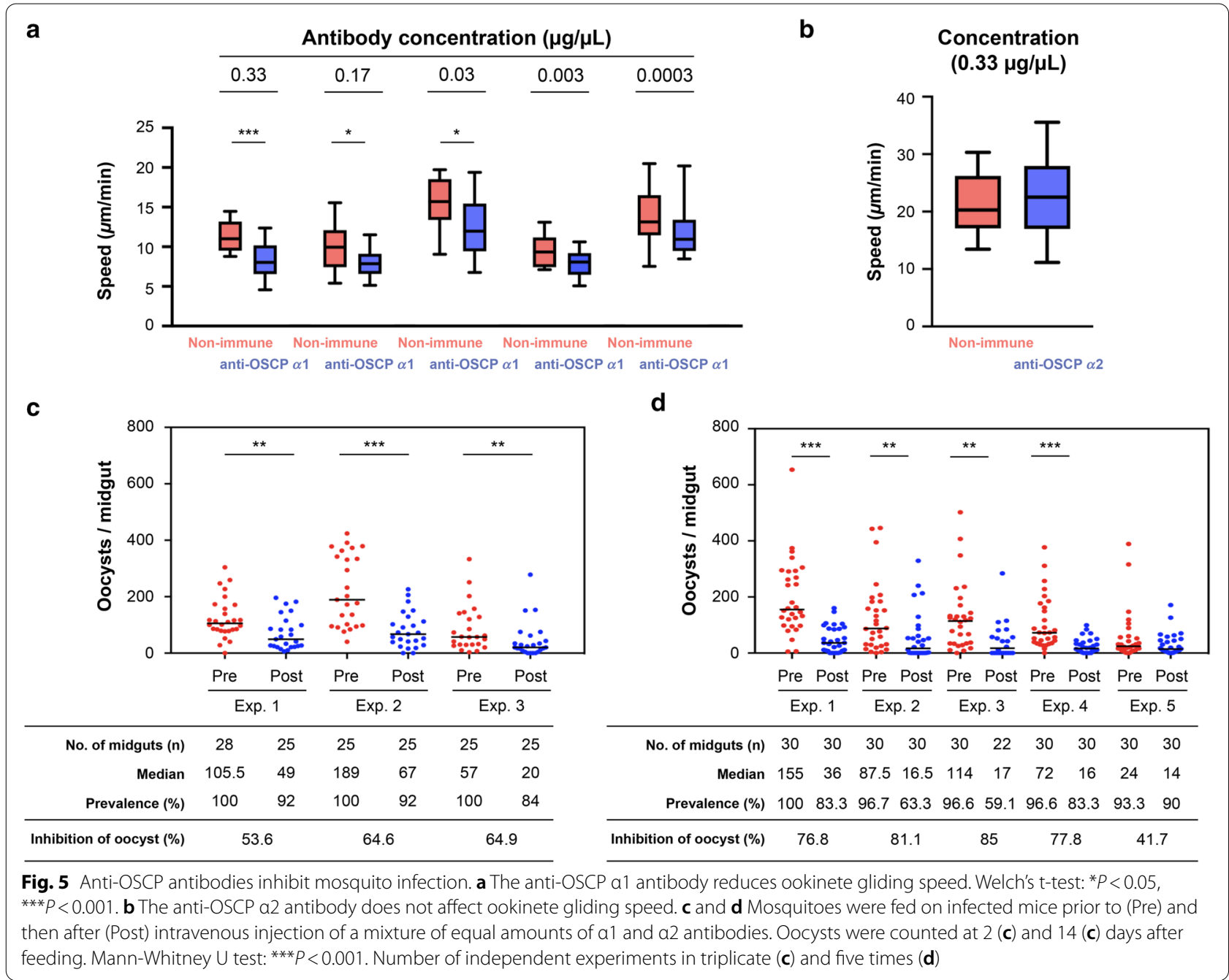

in the midgut tissue before reaching the area below the basal lamina from the midgut lumen. Ookinetes damage the midgut cells while traveling to the basal lamina, which activates the mosquito's immune defenses, causing the ookinete to be eliminated [35]. Accordingly, ookinetes that move in a linear fashion exhibit decreased ability in infecting the mosquito. In fact, one study found that knocking out the IMC1h present below the ookinete cell membrane caused the ookinetes to move in a linear fashion, and the number of oocysts decreased [31]. In this case, because of the reduction in the speed of ookinete movement and the change in morphology, although it is unlikely that the linear motion directly caused the decrease in the number of oocysts, the change in the trajectory of motion could be one of the factors. In our study, the OSCP-KO parasite exhibited decreased movement speed as well as deviation in the normal spiral movement. The decreased number of turns in threedimensional space noted in the KO parasite could have been due to the ookinete movement changing from a spiral to a linear pattern. In an in vivo experiment using mosquitoes, ookinetes that perform spiral movements were found to be involved in invasion below the midgut basal lamina [35]. Our ookinetes performed spiral movements in a three-dimensional space on the Matrigel-coated slide glass. For every two turns, the ookinetes turned once on a spiral-based axis (Fig. 3d). This indicates that OSCP may also be involved in the performance of spiral movements upon invasion of the midgut in vivo. These results therefore suggest that OSCP is involved in ookinete locomotion. Since no changes to ookinete morphology were observed in the OSCP-KO parasite, we inferred that this was because the altered movement trajectories were not due to abnormal adhesion to the extracellular matrix, which is required during ookinete movement. The results of a previous in vivo experiment also observed adhesion to the midgut cells was alongside rotational movement; a similar movement was observed in our experiment's 
response [34, 35]. Moreover, our evaluation focused on spiral movement in order to investigate locomotion to the area under the midgut basal lamina.

Unlike CTRP, however, OSCP is also expressed in the oocyst wall. Five days after ingestion of the blood meal, the number of oocysts decreased and they were smaller in the KO parasite compared to the WT parasite. When the existing oocyst wall structural proteins $\mathrm{PbCap} 380$ and $\mathrm{PbCap} 93$ were knocked out, the number of oocysts that had formed 15 days after the blood meal decreased $[24,25]$. In the PbCap93-KO parasite, oocyst size was also smaller at 15 days after the blood meal. This suggests that OSCP is involved in oocyst growth as a wall structural protein. In the OSCP-KO parasite, the number of oocysts decreased as more days had passed since the blood meal. Accordingly, oocysts that had formed might have quickly been eliminated by the mosquito's immune defenses during the early oocyst stage. Similar results were also observed in the $\mathrm{PbCap} 380-\mathrm{KO}$ parasite. In the OSCP-KO parasite, electron microscope images demonstrated thinning of the oocyst wall and delaying of sporozoite differentiation. It appears that oocyst wall structural proteins function by covering the oocyst to hide it from the mosquito's immune defenses. Therefore, thinning of the oocyst wall causes the oocyst to be recognized by the mosquito's immune defenses, resulting in destruction of the oocyst [25]. Moreover, thinning of the oocyst wall affects differentiation of the sporozoite, with some sporozoites being immature in the KO oocysts. This suggests that OSCP is also involved in oocyst maintenance.

Ookinete movement speed decreased after the $\alpha-1$ antibody was exposed to the 926-939 amino acid part of OSCP. In addition, when mosquitoes ingested infected blood containing anti-OSCP $\alpha-1$ and $\alpha-2$ antibodies, the number of oocysts decreased 2 days after the blood meal, and infection rates also decreased 5 days after the blood meal. These results suggest that anti-OSCP $\alpha-1$ antibodies that recognize the 926-939 amino acid block transmission. Ookinetes travel passively through the midgut lumen until they reach the midgut cell surface. By connecting to the midgut cells, the ookinete is able to exhibit motility and travel to below the midgut basal lamina [36]. This suggests that antibodies in the ingested blood attached to the matured ookinete surface and lowered ookinete-midgut affinity, thereby reducing movement speed and blocking transmission. The changes to the three-dimensional structure of OSCP due to antibody binding appeared to directly affect ookinete motility.

To achieve TB in a parasite, a control using a TB vaccine (TBV) for proteins expressed on the parasite surface in the mosquito stages has been proposed. There are two kinds of TBVs: pre-fertilization TBVs target proteins expressed on female gametocyte and female gamete surfaces, while post-fertilization TBVs target proteins that are expressed on zygote and ookinete surfaces [29, 37]. The anti-OSCP antibodies decreased the gliding speed of WT ookinetes in vitro. Moreover, the anti-OSCP antibodies reduced the number of oocysts 2 days after the blood meal. This implies that OSCP could be a new candidate for a post-fertilization TBV protein.

Previous results have shown that OSCP is involved in ookinete invasion into the midgut cells and in maintaining the structure of the oocyst wall, suggesting that anti-OSCP antibodies would block transmission. Only knocking out OSCP did not cause any major changes to morphology and did not completely stop movement or transmission to mosquitoes. Based on this, it can be predicted that even if OSCP is knocked out, some proteins may function to compensate for its loss. Using antiOSCP antibodies decreased infection rates to mosquitoes by $25.4 \%$ on day 15 after the blood meal. The reason why anti-OSCP antibodies did not completely block transmission may be because of the same reason as for $\mathrm{KO}$ parasites. In case of inhibition of OSCP by antibodies, some proteins may function to compensate for its loss. To achieve a complete blocking efficacy, it is desirable to search for epitopes that have better effects to reduce infection rates of mosquitoes. A previous model has shown that malaria can be eradicated if transmission is blocked by $35 \%$ [38]. Therefore, better epitopes of OSCP and/or its related proteins that can block transmission from mosquitoes by $35 \%$ are required. Going forward, to analyze the mechanism for ookinete motility changes, future studies should address whether complexes had formed and whether interaction with mosquito-derived proteins is seen. Further research also needs to investigate whether anti-OSCP antibodies function similarly in blocking transmission to $P$. falciparum.

\section{Conclusions}

In summary, we identified that the OSCP protein has functions in both the ookinete motility and oocyst growth. In addition, anti-OSCP antibodies reduce ookinete speed and have an inhibitory effect on oocyst formation. Further studies are needed to determine the potential role of the OSCP protein and whether antiOSCP antibodies can be used as a TB tool for malaria.

\footnotetext{
Abbreviations

CelTos: Cell-traversal protein for ookinetes and sporozoites; CTRP: Circumsporozoite- and TRAP-related protein; GCB: Guanylate cyclase $\beta$; IFA: Immunofluorescence assays; KO: Knockout; LYSC1: Lysozyme c-1; MMP1: Matrix metalloprotease 1; OKM: Complete ookinete medium; OSCP: Ookinete surface and oocyst capsule protein; PbCap380: Plasmodium berghei Oocyst capsule
} 
protein 380; PbCap93: Oocyst capsule-associated protein 93 of Plasmodium berghei; PIMMS2: Plasmodium Invasion of mosquito midgut screen candidate 2; PPLP3-5: Plasmodium Perforin-like protein 3-5; PM: Peritrophic matrix; RT: Room temperature; SOAP: Secreted ookinete adhesive protein; TB: Transmission blocking; TBV: Transmission-blocking vaccine; WT: Wild type.

\section{Acknowledgements}

The Plasmodium berghei ANKA strain and anti-PbCap380 antibody used in this work were kindly provided by Dr. Marcelo Jacobs-Lorena, Johns Hopkins University, MD, USA. The P. berghei ANKA strain that expresses GFP was provided by Dr. Yuda, Mie University, Japan. The Anopheles stephensi (STE2 strain, MRA-128) was provided by BEI Resources. This study was supported by the JSPS KAKENHI Grant Number 17H04073 (to HI) and 16H05026, 19K22355 (to SF), and the Cooperative Research Grant (29-joint-12, 30-joint-10, 2019-joint-6) of the National Research Center for Protozoan Diseases, Obihiro University of Agriculture and Veterinary Medicine (to HI). We thank Dr. Marcelo JacobsLorena for engaging in constructive discussions with us on particular aspects of this work. This work would not have been possible without the assistance of Mai Tanaka, a support staff in our laboratory.

\section{Permission to reproduce material from other sources}

No third-party material requiring formal permission has been used in this study.

\section{Authors' contributions}

$\mathrm{KN}$ and $\mathrm{HI}$ have contributed toward conception and design of the study, and drafting the manuscript. KN, Y Kimura, Y Kitahara, AS, AH, JH, KK, and $\mathrm{HI}$ have contributed toward acquisition of data. KN, Y Kimura, KK, SF, and $\mathrm{HI}$ have performed the analyses and interpretation of data. $\mathrm{KN}$ and $\mathrm{HI}$ were involved in critical revision of the manuscript for important intellectual content. KN, $Y$ Kimura, and Y Kitahara did the statistical analyses of the data. Y Kimura, Y Kitahara, and MS contributed toward electron microscopy analyses. KN, Y Kimura, Y Kitahara, $\mathrm{AS}, \mathrm{AH}, \mathrm{JH}, \mathrm{KK}, \mathrm{SF}$, and $\mathrm{HI}$ provided technical or material support $\mathrm{HI}$ supervised the study. All authors read and approved the final manuscript.

\section{Funding}

This study was supported by the JSPS KAKENHI Grant Number 17 H04073 (to $\mathrm{HI}$ ) and 16H05026, $19 \mathrm{~K} 22355$ (to SF), and the Cooperative Research Grant (29-joint-12, 30-joint-10, 2019-joint-6) of the National Research Center for Protozoan Diseases, Obihiro University of Agriculture and Veterinary Medicine (to HI).

\section{Availability of data and materials}

Data available on request from the authors.

\section{Declarations}

\section{Ethics approval and consent to participate}

All procedures were executed in accordance with a protocol approved by the Kitasato University Animal Care and Use Committee (nos. 18-029 and 18-030). Consent for participation was not required in this study.

\section{Consent for publication}

Not applicable.

\section{Competing interests}

The authors declare that they have no competing interests.

\section{Author details}

'Laboratory of Veterinary Parasitology, School of Veterinary Medicine, Kitasato University, Towada, Aomori 034-8628, Japan. ${ }^{2}$ National Research Center for Protozoan Diseases, Obihiro University of Agriculture and Veterinary Medicine, Inada, Obihiro 080-8555, Japan. ${ }^{3}$ Laboratory of Veterinary Anatomy, School of Veterinary Medicine, Kitasato University, Towada, Aomori 034-8628, Japan

Received: 13 March 2021 Accepted: 30 June 2021

Published online: 21 July 2021

\section{References}

1. World Health Organization. Malaria. https://www.who.int/news-room/ fact-sheets/detail/malaria. Accessed 26 May 2021.

2. Bennink S, Kiesow MJ, Pradel G. The development of malaria parasites in the mosquito midgut. Cell Microbiol. 2016;18:905-18. https://doi.org/10. 1111/cmi.12604.

3. Angrisano F, Tan YH, Sturm A, McFadden Gl, Baum J. Malaria parasite colonisation of the mosquito midgut - placing the Plasmodium ookinete centre stage. Int J Parasitol. 2012;4:519-27. https://doi.org/10.1016/j. ijpara.2012.02.004.

4. Smith RC, Vega-Rodríguez J, Jacobs-Lorena M. The Plasmodium bottleneck: malaria parasite losses in the mosquito vector. Mem Inst Oswaldo Cruz. 2014;109:644-61. https://doi.org/10.1590/0074-0276130597.

5. Ghosh A, Edwards MJ, Jacobs-Lorena M. The journey of the malaria parasite in the mosquito: hopes for the new century. Parasitol Today. 2000;16:196-201. https://doi.org/10.1016/s0169-4758(99)01626-9.

6. Han YS, Thompson J, Kafatos FC, Barillas-Mury C. Molecular interactions between Anopheles stephensi midgut cells and Plasmodium berghei: the time bomb theory of ookinete invasion of mosquitoes. EMBO J. 2000;19:6030-40. https://doi.org/10.1093/emboj/19.22.6030.

7. Kumar S, Barillas-Mury C. Ookinete-induced midgut peroxidases detonate the time bomb in anopheline mosquitoes. Insect Biochem Mol Biol. 2005;35(7):721-7. https://doi.org/10.1016/j.ibmb.2005.02.014.

8. Liu F, Li L, Zheng W, He Y, Wang Y, Zhu X, et al. Characterization of Plasmodium berghei $\mathrm{Pbg} 37$ as both a pre- and postfertilization antigen with transmission-blocking potential. Infect Immun. 2018;86:e00785-e817. https://doi.org/10.1128/IAl.00785-17.

9. Tomas AM, Margos G, Dimopoulos G, van Lin LHM, de Koning-Ward TF, Sinha R, et al. P25 and P28 proteins of the malaria ookinete surface have multiple and partially redundant functions. EMBO J. 2001;20:3975-83. https://doi.org/10.1093/emboj/20.15.3975.

10. Ukegbu CV, Akinosoglou KA, Christophides GK, Vlachou D. Plasmodium berghei PIMMS2 promotes ookinete invasion of the Anopheles gambiae mosquito midgut. Infect Immun. 2017;85:e00139-e217. https://doi.org/ 10.1128/IAI.00139-17.

11. Ukegbu CV, Giorgalli M, Tapanelli S, Rona LDP, Jaye A, Wyer C, et al. PIMMS43 is required for malaria parasite immune evasion and sporogonic development in the mosquito vector. Proc Natl Acad Sci U S A. 2020;117:7363-73. https://doi.org/10.1073/pnas.1919709117.

12. Ukegbu CV, Giorgalli M, Yassine H, Ramirez JL, Taxiarchi C, Barillas-Mury C, et al. Plasmodium berghei P47 is essential for ookinete protection from the Anopheles gambiae complement-like response. Sci Rep. 2017;7:6026. https://doi.org/10.1038/s41598-017-05917-6.

13. Deligianni E, Silmon de MNCS, McMillan PJ, Bertuccini L, Superti F, Manola $M_{1}$, et al. Essential role of Plasmodium perforin-like protein in ookinete midgut passage. PLOS ONE. 2018;13:e0201651. https://doi.org/10.1371/ journal.pone.0201651.

14. Dessens JT, Beetsma AL, Dimopoulos G, Wengelnik K, Crisanti A, Kafatos FC, Sinden RE. CTRP is essential for mosquito infection by malaria ookinetes. EMBO J. 1999;18:6221-7. https://doi.org/10.1093/emboj/18.22.6221.

15. Dessens JT, Mendoza J, Claudianos C, Vinetz JM, Khater E, Hassard S, et al. Knockout of the rodent malaria parasite chitinase pbCHT1 reduces infectivity to mosquitoes. Infect Immun. 2001;69:4041-7. https://doi.org/ 10.1128/IAI.69.6.4041-4047.2001.

16. Dessens JT, Sidén-Kiamos I, Mendoza J, Mahairaki V, Khater E, Vlachou D, et al. SOAP, a novel malaria ookinete protein involved in mosquito midgut invasion and oocyst development. Mol Microbiol. 2003;49:319-29. https://doi.org/10.1046/j.1365-2958.2003.03566.x.

17. Ecker A, Pinto SB, Baker KW, Kafatos FC, Sinden RE. Plasmodium berghei: Plasmodium perforin-like protein 5 is required for mosquito midgut invasion in Anopheles stephensi. Exp Parasitol. 2007;1 16:504-8. https://doi.org/ 10.1016/j.exppara.2007.01.015.

18. Kadota K, Ishino T, Matsuyama T, Chinzei Y, Yuda M. Essential role of membrane-attack protein in malarial transmission to mosquito host. Proc Natl Acad Sci U S A. 2004;101:16310-5. https://doi.org/10.1073/pnas. 0406187101

19. Kaneko I, Iwanaga S, Kato T, Kobayashi I, Yuda M. Genome-wide identification of the target genes of AP2-O, a Plasmodium AP2-Family transcription factor. PLOS Pathog. 2015;11: e1004905. https://doi.org/10.1371/journal. ppat.1004905. 
20. Kariu T, Ishino T, Yano K, Chinzei Y, Yuda M. CelTOS, a novel malarial protein that mediates transmission to mosquito and vertebrate hosts. Mol Microbiol. 2006;59(5):1369-79. https://doi.org/10.1111/j.1365-2958.2005. 05024.x.

21. Tsai YL, Hayward RE, Langer RC, Fidock DA, Vinetz JM. Disruption of Plasmodium falciparum chitinase markedly impairs parasite invasion of mosquito midgut. Infect Immun. 2001;69(6):4048-54. https://doi.org/10. 1128/IAl.69.6.4048-4054.2001.

22. Yuda M, Sakaida H, Chinzei Y. Targeted disruption of the Plasmodium berghei CTRP gene reveals its essential role in malaria infection of the vector mosquito. J Exp Med. 1999;190:1711-6. https://doi.org/10.1084/jem. 190.11.1711.

23. Smith RC, Barillas-Mury C. Plasmodium oocysts: overlooked targets of mosquito immunity. Trends Parasitol. 2016;32:979-90. https://doi.org/10. 1016/j.pt.2016.08.012.

24. Sasaki H, Sekiguchi H, Sugiyama M, Ikadai H. Plasmodium berghei Cap93, a novel oocyst capsule-associated protein, plays a role in sporozoite development. Parasites Vectors. 2017;10:399. https://doi.org/10.1186/ s13071-017-2337-8.

25. Srinivasan P, Fujioka H, Jacobs-Lorena M. PbCap380, a novel oocyst capsule protein, is essential for malaria parasite survival in the mosquito. Cell Microbiol. 2008;10:1304-12. https://doi.org/10.1111/j.1462-5822.2008. 01127.x.

26. Ishino T, Orito Y, Chinzei Y, Yuda M. A calcium-dependent protein kinase regulates Plasmodium ookinete access to the midgut epithelial cell. Mol Microbiol. 2006;59:1175-84. https://doi.org/10.1111/j.1365-2958.2005. 05014.x.

27. Janse CJ, Ramesar J, Waters AP. High-efficiency transfection and drug selection of genetically transformed blood stages of the rodent malaria parasite Plasmodium berghei. Nat Protoc. 2006;1:346-56. https://doi.org/ 10.1038/nprot.2006.53.

28. Yuda M, Iwanaga S, Shigenobu S, Kato T, Kaneko I. Transcription factor AP2-Sp and its target genes in malarial sporozoites. Mol Microbiol. 2010;75:854-63. https://doi.org/10.1111/j.1365-2958.2009.07005.x.

29. Saxena AK, Wu Y, Garboczi DN. Plasmodium p25 and p28 surface proteins: potential transmission-blocking vaccines. Eukaryot Cell. 2007;6:1260-5. https://doi.org/10.1128/EC.00060-07.

30. Carter V, Cable HC, Underhill BA, Williams J, Hurd H. Isolation of Plasmodium berghei ookinetes in culture using Nycodenz density gradient columns and magnetic isolation. Malar J. 2003;2:35. https://doi.org/10. 1186/1475-2875-2-35

31. Volkmann K, Pfander C, Burstroem C, Ahras M, Goulding D, Rayner JC, et al. The alveolin IMC $1 \mathrm{~h}$ is required for normal ookinete and sporozoite motility behaviour and host colonisation in Plasmodium berghei. PLoS ONE. 2012;7: e41409. https://doi.org/10.1371/journal.pone.0041409.

32. Usui M, Fukumoto S, Inoue N, Kawazu S. Improvement of the observational method for Plasmodium berghei oocysts in the midgut of mosquitoes. Parasit Vectors. 2011;4:118. https://doi.org/10.1186/ 1756-3305-4-118.

33. Kan A, Tan YH, Angrisano F, Hanssen E, Rogers KL, Whitehead L, et al. Quantitative analysis of Plasmodium ookinete motion in three dimensions suggests a critical role for cell shape in the biomechanics of malaria parasite gliding motility. Cell Microbiol. 2014;16:734-50. https://doi.org/ 10.1111/cmi.12283.

34. Trisnadi N, BarillasMury C. Live in vivo imaging of Plasmodium invasion of the mosquito midgut. mSphere. 2020;5:0e00692-20. https://doi.org/10. 1128/mSphere.00692-20

35. Vlachou D, Zimmermann T, Cantera R, Janse CJ, Waters AP, Kafatos FC. Real-time, in vivo analysis of malaria ookinete locomotion and mosquito midgut invasion. Cell Microbiol. 2004;6:671-85. https://doi.org/10.1111/j. 1462-5822.2004.00394.x.

36. Douglas RG, Amino R, Sinnis P, Frischknecht F. Active migration and passive transport of malaria parasites. Trends Parasitol. 2015;31:357-62. https://doi.org/10.1016/j.pt.2015.04.010.

37. De Jong RM, Tebeje SK, Meerstein-Kessel L, Tadesse FG, Jore MM, Stone W et al. Immunity against sexual stage Plasmodium falciparum and Plasmodium vivax parasites. Immunol Res. 2020;293:190-215. https://doi.org/10. 1111/imr.12828.

38. Blagborough AM, Churcher TS, Upton LM, Ghani AC, Gething PW, Sinden RE. Transmission-blocking interventions eliminate malaria from laboratory populations. Nat Commun. 2013;4:1812. https://doi.org/10.1038/ ncomms 2840 .

\section{Publisher's Note}

Springer Nature remains neutral with regard to jurisdictional claims in published maps and institutional affiliations.
Ready to submit your research? Choose BMC and benefit from:

- fast, convenient online submission

- thorough peer review by experienced researchers in your field

- rapid publication on acceptance

- support for research data, including large and complex data types

- gold Open Access which fosters wider collaboration and increased citations

- maximum visibility for your research: over 100M website views per year

At BMC, research is always in progress.

Learn more biomedcentral.com/submissions 